\section{Assessment of Thyroid Lobe Dose in Breast Cancer Intraoperative Radiotherapy}

\author{
Ramezani Farkhani R. ${ }^{1 \oplus}$, Gholamhosseinian H. ${ }^{2 *}$, Anvari \\ K. ${ }^{3}$, Forghani M. N. ${ }^{4}$
}

\begin{abstract}
Background: Breast cancer is the most common cancer among women. Considering the fact that a high dose is delivered in a single fraction of IORT, the evaluation of the dose at sensitive organs like thyroid is necessary.
\end{abstract}

Objective: The current study has aimed to evaluate the received dose to thyroid lobes in the breast IORT technique.

Material and Methods: A total of 49 women with breast cancer undergoing IORT were enrolled in this cross-sectional study with census sampling. Immediately after tumor resection, a single dose of 20 Gray at the applicator surface was delivered using $50 \mathrm{KV} \mathrm{X}$-ray by an Intrabeam machine. The thyroid dose was detected using thermoluminescent detectors (TLD) 100 at the mid-thyroid line, left and right lobes.

Results: The dose at the right and left lobes of the thyroid gland as well as the mid-thyroid line was found to be $40.18 \pm 35.44 \mathrm{mGy}, 35.50 \pm 27.32 \mathrm{mGy}$, and $40.61 \pm 32.47 \mathrm{mGy}$, respectively. The right lobe received a significantly higher absorbed dose compared to the left lobe when the right breast was under IORT treatment. The same trend was seen with the left lobe and left breast under IORT treatment $(\mathrm{P}=0.0001$ and $\mathrm{P}=0.018$, respectively). The applicator size showed non-significant effects on the absorbed dose at the thyroid gland. Also, the applicator depth had a non-significant inverse effect on thyroid dose.

Conclusion: According to our findings, the absorbed dose at each thyroid lobe depends on the under-treatment side as well as the applicator size and depth (applicator upper surface distance from the skin).

Citation: Ramezani Farkhani R, Gholamhosseinian H, Anvari K, Forghani MN. Assessment of Thyroid Lobe Dose in Breast Cancer Intraoperative Radiotherapy. J Biomed Phys Eng. 2021;11(1):55-60. doi: 10.31661/jbpe.v0i0.2001-1046.

\section{Keywords}

Breast Cancer; Intraoperative Radiotherapy; Thyroid; Radiotherapy Dosage; Thermoluminescent Dosimetry

\section{Introduction}

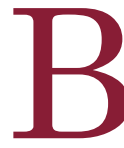

reast cancer is one of the most common types of cancer among women throughout the world [1]. Cancer treatment involves surgery, chemotherapy, hormone therapy, and radiotherapy [2]. Intraoperative radiotherapy (IORT) is one of the specific methods of radiotherapy in which a defined dose of radiation is delivered into the tumor bed in a single fraction of treatment [3]. Low-energy (30-50 KV) $\mathrm{X}$-ray IORT is an innovative method to improve the treatment effects in patients with breast cancer. The tumor bed can be treated using a
${ }^{1} \mathrm{MSc}$, Department of Medical Physics, Faculty of Medicine, Mashhad University of Medical Sciences, Mashhad, Iran ${ }^{2} \mathrm{PhD}$, Department of Medical Physics, Faculty of Medicine, Mashhad University of Medical Sciences, Mashhad, Iran

${ }^{3} \mathrm{MD}$, Department of Radiotherapy \& Oncology, Omid Hospital, Mashhad University of Medical Sciences, Mashhad, Iran ${ }^{4} \mathrm{MD}$, Department of General Surgery, Mashhad University of Medical Sciences, Mashhad, Iran

*Corresponding author: H. Gholamhosseinian Department of Medical Physics, Faculty of Medicine, Mashhad University of Medical Sciences, Mashhad, Iran

E-mail: gholamhosseinianh@mums.ac.ir

Received: 7 January 2020 Accepted: 8 March 2020 
single high-dose of X-ray immediately after surgical resection of the tumor. Considering the significant reduction in the dermal side effects compared to the external radiotherapy, this technique is highly superior from the cosmetic point of view. Also, single dose delivery decreases the treatment time and costs [4].

For those patients with breast cancer recurrence who have been treated once before, the breast tissue will not tolerate irradiation after the second surgery and hence, total mastectomy is suggested. Partial breast irradiation during surgery is a new approach for these patients. It is believed that this method increases the effectiveness of restricted local re-irradiation while the side effects are acceptable [5]. The skin and subdermal tissues are not irradiated, resulting in a lower risk of fibrosis and a higher efficacy with IORT [6].

Considering the fact that a high dose is delivered in a single fraction of treatment, the evaluation of the radiation dose at the sensitive organs like thyroid is necessary. The absorbed dose has been claimed to be associated with thyroid damage and cancer [7-9].

The current research provides a reliable method for the measurement of the ionizing radiation at the thyroid lobes and suggests a correlation between the dose at the thyroid lobes and the breast under treatment using TLDs at defined distances on the thyroid skin in 49 patients.

Due to the higher electron density of the TLDs compared to water, the $0.9 \mathrm{~mm}$ thickness of the TLDs is assumed as $1 \mathrm{~mm}$ [10].

\section{Material and Methods}

A total of 49 women with breast cancer undergoing IORT were enrolled in this crosssectional study with census sampling. An IRTABEAM $^{\circledR}$ (ZEISS) machine containing an X-ray probe and a control unit (Photon Radiosurgery System, PRS) along with a series of spherical applicators with the diameter ranges of $1.5-5 \mathrm{~cm}$ were used in this research. The applicator size was chosen considering the size of the void from tumor resection. The applicator was placed directly onto the tumor bed (Figure 1: A-C). A homogeneous beam of 50 $\mathrm{Kv}$ was produced at the probe tip. A $20 \mathrm{~Gy}$ dose was locally administered at the applicator surface to the tumor bed. The duration of treatment depended on the applicator size. A minimums distance of $10 \mathrm{~mm}$ was created between the skin and the applicator surface using wet sterile gauzes when necessary [11].

Considering the tissue inhomogeneity around the treatment site, in vivo dosimetry provides a precise and accurate method to ensure the quality of measurement at the sensitive thyroid tissue. TLD dosimeters are suitable tools for this type of measurement due to their small sizes [12]. Dose measurement at the thyroid lobes was performed using three TLD-100s in $2 \mathrm{~cm}$ distances from each other. Immediately after tumor resection, a single dose of 20 Gray at the applicator surface was delivered using $50 \mathrm{KV}$ X-ray by an Intrabeam machine. The applicator size is selected according to the resected tumor size. The diameters of these applicators range from $1.5-5 \mathrm{~cm}$ in $0.5 \mathrm{~cm}$ intervals. The duration of treatment depends on the applicator size as well as the delivered dose and it is between 8 and $45 \mathrm{~min}$ for a dose of $20 \mathrm{~Gy}$. Sonography and mammography images help to make a decision on the proper size of the applicator as well as estimating the distance from the applicator and the margins of the surgical incision.

A set of three Lithium-Fluoride TLDs (Dimensions: $3.2 \times 3.2 \times 0.9 \mathrm{~mm}$ ) was placed at the left and right lobes as well as the mid-thyroid line with $2 \mathrm{~cm}$ distance from each other (Figures 1: D and E). The TLDs were calibrated using $50 \mathrm{KV}$ energy on an INTRABEAM apparatus before use. For the first step, the element correction coefficient (ECC) was calculated and then, the reader correction factor (RCF) was calculated from the reader machine. For the third step, the RCF constant for the Harshaw 3500 TLD reader was calculated using the TLD reader Vinerm software. The fi- 

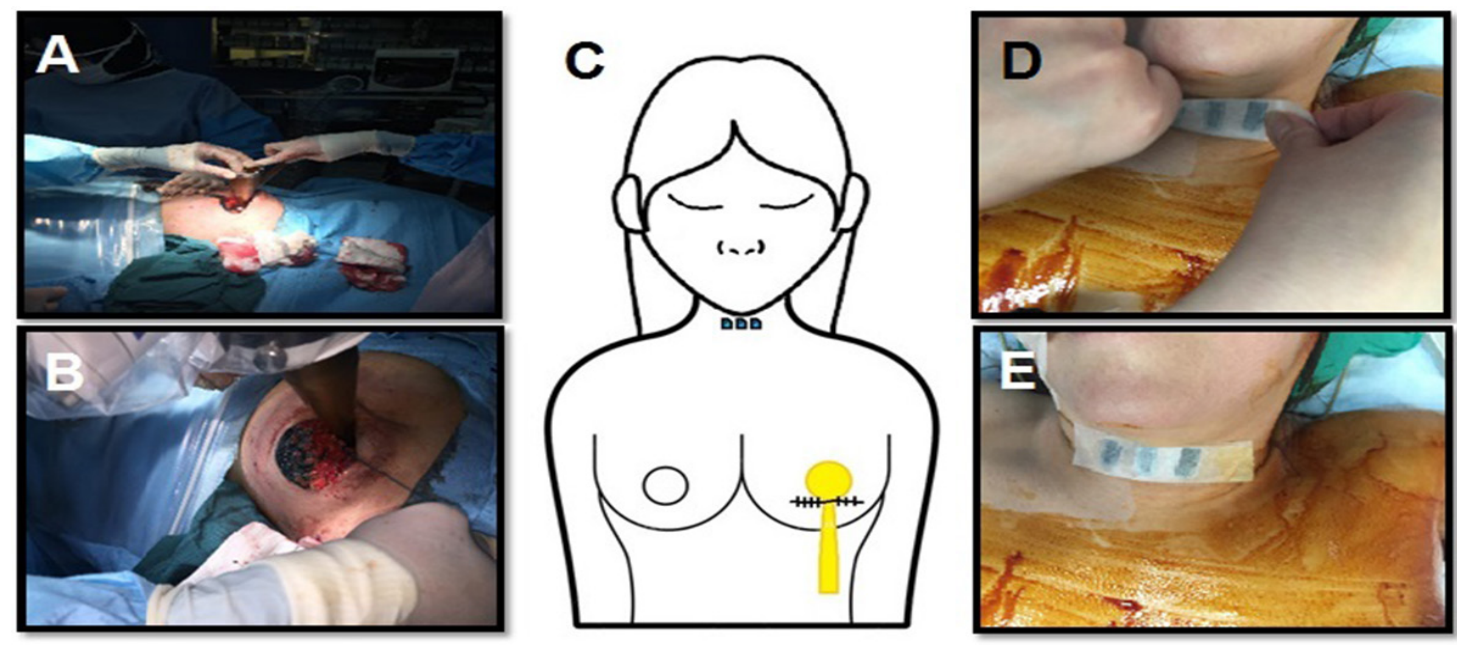

Figure 1: The locations of A-C) Applicator and C-E) thermoluminescent detectors on the left and right lobes of the thyroid as well as the mid-thyroid line.

nal step was calibrating the ECC for all TLDs.

The TLDs were annealed before irradiation to confirm the release of the residual dose from previous irradiations. The annealing process was performed using and Ex.1200-12s electrical furnace $\left(\right.$ Exciton $\left.^{\circledR}\right)$ at $400{ }^{\circ} \mathrm{C}$ for 1 $\mathrm{h}$ followed by $10 \mathrm{~min}$ incubation at room temperature and another $100{ }^{\circ} \mathrm{C}$ treatment for 2 $\mathrm{h}$. The annealing was performed at least a day before irradiation.

After irradiation, the TLDs were read by a reader and the values were converted to dose.

\section{Results}

The dose at the right and left lobes of the thyroid gland as well as the mid-thyroid line was found to be $40.18 \pm 35.44$ mGy (Max: 135.82 mGy, Min: 4.57 mGy), 35.50 27.32 mGy (Max: 118.62 mGy, Min: $5.55 \mathrm{mGy}$ ), and 40.61 $\pm 32.47 \mathrm{mGy}$ (Max: $132.23 \mathrm{mGy}$, Min: $8.41 \mathrm{mGy}$ ), respectively.

Student t-test showed that when the right breast was under IORT treatment, the right lobe of the thyroid gland received a significantly higher dose of ionizing radiation compared to the left lobe; when the left breast was under treatment, the left lobe of the thyroid absorbed a significantly higher dose of ionizing radiation compared to the right lobe, however, the difference was not statistically significant at the mid-thyroid line with any of the left or right breasts under IORT (Figure 2).

Multivariate linear regression showed that increasing the applicator size showed an increasing trend on the dose detected by TLDs at the thyroid gland (Figure 3).

Multivariate linear regression showed that increasing the applicator upper surface distance from the skin showed a decreasing trend on the dose detected by TLDs at the thyroid gland (Figure 4).

Based on the position of the tumor that we have considered a circle at the center of the nipple, the tumor was placed in one of the four positions of the inner and outer upper quadrant and the inner and lower quadrant. To illustrate the effect of tumor location on the thyroid received dose, we measured the right and left lobes of thyroid with the upper-inner quadrant and lower-lateral quadrant of ipsilateral breast. Mean dose of right-sided thyroid lobe in right treated breast in two positions of the internal upper quadrant $(78.3 \pm 37.73 \mathrm{mGy})$ and external lower quadrant $(48.24 \pm 26.38 \mathrm{mGy})$ and in left-sided thyroid lobe with left treated breast in two positions of the inner-upper quadrant 


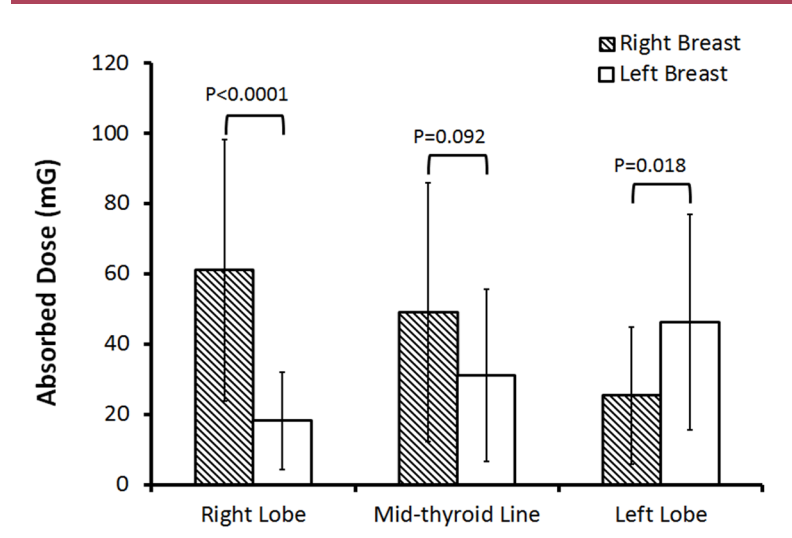

Figure 2: The dose at each thyroid lobe when each of the right or left breasts is under IORT treatment.

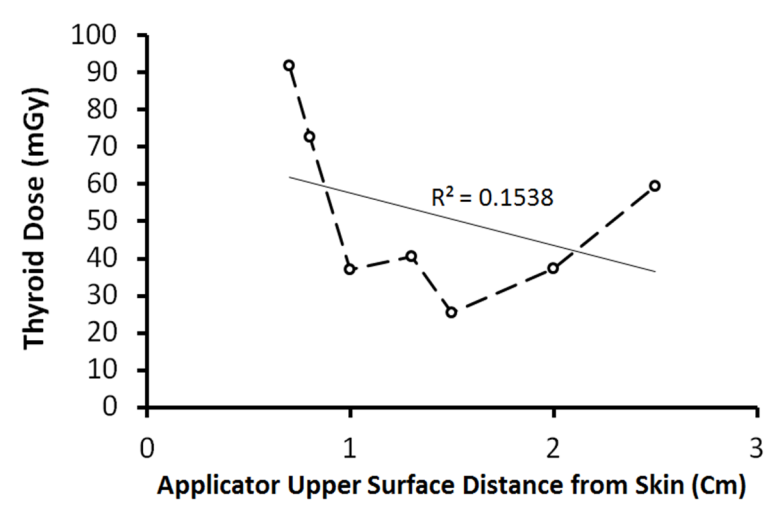

Figure 4: The relationship between the applicator depth and dose detected at each lobe of the thyroid gland as well as the midthyroid line.

$(60.5 \pm 43.34 \mathrm{mGy})$ and the outer-lower quadrant $(26.79 \pm 8.51 \mathrm{mGy})$ was obtained. As was expected, the applicator position had an effect on the thyroid dose received and when the position was near the thyroid, it received a higher dose (Figure 5).

\section{Discussion}

A major disadvantage of common radiotherapy techniques is the received dose to healthy tissues and sensitive organs. During radio-

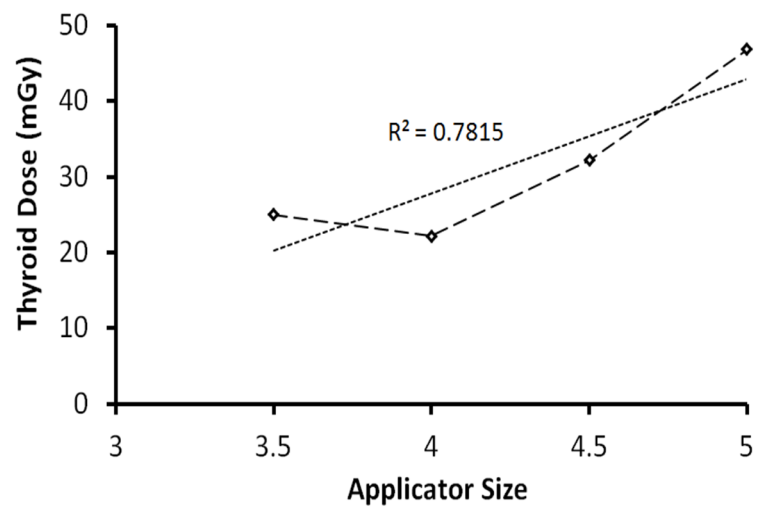

Figure 3: The relationship between the applicator size and the dose detected at each lobe of the thyroid gland as well as the midthyroid line

therapy for breast cancer treatment, a number of sensitive tissues are affected and injured as well. When performing an IORT, a high dose of irradiation is delivered to the target organ in a single fraction of treatment, and hence, in vivo dosimetry for sensitive organs and tissues is very important. The received dose to the thyroid gland in the breast cancer IORT technique has never been reported so far. In this research project, we used TLD-100 to measure the received dose to each thyroid lobe as well as the mid-thyroid line. Our results showed that the dose each thyroid lobe receives is as low as $\sim 40 \mathrm{mGy}$. Moreover, the breast under IORT is a determining factor in the received dose to each lobe. Also, the bigger the applicator size is, the higher the thyroid dose will be. Moreover, the applicator depth (applicator upper surface distance from the skin) has a slight negative impact on the dose detected at the thyroid gland.

Although all available patients undergoing breast IORT were enrolled during this study, as a limitation, the sample size to make a general deduction to reach a mathematical formula seems not to be enough yet. Also, the anatomical diversity in the breast size and volume, as well as the anthropometric indexes in the participants of this study has caused wide 

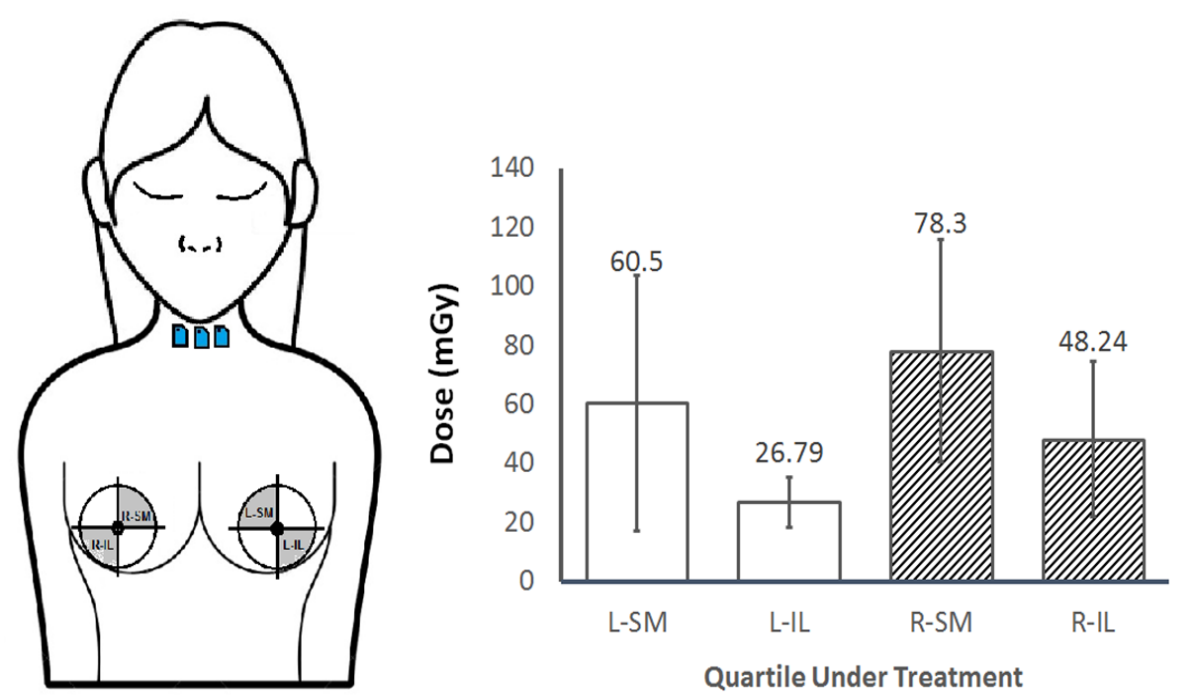

Figure 5: The mean absorbed dose in left and right thyroid lobes based on inner-upper and outer-lower quadrants of the left and right breasts (L-SM=Left breast, Superomedial; L-IL=Left breast, Inferolateral; R-SM=Right breast, Superomedial; R-IL=Right breast, Inferolateral).

ranges of data resulting in big standard deviations.

The effect of the breast side under IORT treatment on the dose detected at thyroid gland has never been reported in previous studies. In this study, we showed that the dose detected by each thyroid lobe is significantly depending on the side of IORT. This finding is expected as the distance from each side to the corresponding lobe is lower and hence the dose at that lobe will be higher.

Also, as it was expected, our result confirmed that the bigger the applicator size is, the higher the detected dose of the thyroid gland will be. However, the applicator depth did not show any significant effects on the detected dose at the thyroid gland, although the applicator distance from skin showed a nonsignificant negative effect on the detected dose.

\section{Conclusion}

According to our findings, the dose received by each thyroid lobe in breast cancer IORT is as low as 40.18 $\pm 35.44 \mathrm{mGy}$. This dose in each lobe depends on the breast side under treat- ment, the applicator size, and at least to some extent, to the applicator depth. Further studies with different detectors like film dosimeters; bigger sample size, various organs under IORT especially at the facial area and etc. are suggested to confirm the outcomes of the current study.

\section{Acknowledgment}

The authors would like to acknowledge the financial support from the Mashhad University of Medical Sciences (\#951054-31/03/1396), Mashhad, Iran.

\section{Conflict of Interest}

None

\section{References}

1. Brady LW, Perez CA, Wazer DE. Perez \& Brady's principles and practice of radiation oncology. Lippincott Williams \& Wilkins; 2013.

2. Bellon JR, Come SE, Gelman RS, Henderson IC, Shulman LN, et al. Sequencing of chemotherapy and radiation therapy in early-stage breast cancer: updated results of a prospective randomized trial. Journal of Clinical Oncology. 2005;23(9):1934-40. 
3. Wood WC, Shipley WU, Gunderson LL, Cohen AM, Nardi GL. Intraoperative irradiation for unresectable pancreatic carcinoma. Cancer. 1982;49(6):1272-5. doi: $\quad 10.1002 / 1097-0142(19820315) 49: 6<1272$. PubMed PMID: 6800633.

4. Kraus-Tiefenbacher U, Scheda A, Steil V, Hermann B, Kehrer T, Bauer L, Melchert F, Wenz F. Intraoperative radiotherapy (IORT) for breast cancer using the intrabeam ${ }^{\mathrm{TM}}$ system. Tumori Journal. 2005;91(4):339-45. doi: $10.1177 / 030089160509100411$.

5. Kraus-Tiefenbacher U, Bauer L, Scheda A, Schoeber C, Schaefer J, Steil V, Wenz F. Intraoperative radiotherapy (IORT) is an option for patients with localized breast recurrences after previous externalbeam radiotherapy. BMC Cancer. 2007;7(1):178. doi: 10.1186/1471-2407-7-178.

6. Gatzemeier W, Orecchia R, Gatti G, Intra M, Veronesi $U$. Intraoperative radiotherapy (IORT) in treatment of breast carcinoma--a new therapeutic alternative within the scope of breast-saving therapy? Current status and future prospects. Report of experiences from the European Institute of Oncology (EIO), Mailand. Strahlenther Onkol. 2001;177(7):330-7. doi: 10.1007/pl00002415. PubMed PMID: 11505618.

7. Richardson DB. Exposure to Lonizing Radiation in
Adulthood and Thyroid Cancer Incidence. Epidemiology. 2009;20(2):181-7.

8. Shore RE. Issues and epidemiological evidence regarding radiation-induced thyroid cancer. Radiation Research. 1992;131(1):98-111. doi: $10.2307 / 3578322$.

9. Schneider AB, Ron EL, Lubin J, Stovall M, Gierlowski TC. Dose-response relationships for radiation-induced thyroid cancer and thyroid nodules: evidence for the prolonged effects of radiation on the thyroid. The Journal of Clinical Endocrinology \& Metabolism. 1993;77(2):362-9. doi: 10.1210/ jcem.77.2.8345040.

10. International Atomic Energy Agency . Absorbed Dose Determination in External Beam Radiotherapy. Technical Report Series No. 398; Vienna: IAEA; 2000.

11. Fogg P, Das KR, Kron T, Fox C, Chua B, Hagekyriakou J. Thermoluminescence dosimetry for skin dose assessment during intraoperative radiotherapy for early breast cancer. Australasian Physical \& Engineering Sciences in Medicine. 2010;33(2):211-4. doi: 10.1007/s13246-010-0019-3.

12. Kron T, Butson M, Hunt F, Denham J. TLD extrapolation for skin dose determination in vivo. Radiotherapy and oncology. 1996;41(2):119-23. 